\title{
BENIGN INFANTILE CONVULSIONS WITH GASTROENTERITIS
}

The clinical features of benign convulsions with mild gastroenteritis (CwG) were studied retrospectively in 62 patients hospitalized (Jan 2000-March 2006) at St Mary's Hospital, Kurume City, Japan. Viral infections confirmed in $71 \%$ of fecal specimens were rotavirus in 30 patients, norovirus in 9 , sapovirus in 2, adenovirus in 2, and coxsackievirus A4 in 1. Age of patients with norovirus $(16.7+/-2.7$ months) was significantly younger than those with rotavirus $(23+/-8.7$ months). Duration of seizures due to norovirus $(11.8+/-12 \mathrm{~h})$ was significantly longer than that caused by rotavirus infection $(4.9+/-5.7 \mathrm{~h})$. Blood chemistries, including electrolytes, glucose, and liver function, were similar for the 2 viruses. In 8 patients with CwG due to norovirus, treatment with the anticonvulsant carbamazepine (serum level $5.4+/-1.1 \mathrm{mg} / \mathrm{dl}$ ) significantly shortened duration of seizures as compared to treatment with phenobarbital. In 26 patients with $\mathrm{CwG}$ due to rotavirus, duration of seizures was not correlated with the type of anticonvulsant used. (Kawano G, Oshige K, Syutou S et al. Benign infantile convulsions associated with mild gastroenteritis: a retrospective study of 39 cases including virological tests and efficacy of anticonvulsants. Brain Dev Nov 2007;29:617-622). (Respond: Dr Toyojiro Matsuishi: E-mail: tmatsu(cimed.kurume-u.ac.jp).

COMMENT. Benign infantile convulsions with gastroenteritis, first described in Japan in 1982, occur in infants 6 months to 3 years of age. They are afebrile, repetitive or in clusters, associated with mild dehydration, and rotavirus antigen is frequently detected in stools. The interictal EEG is normal, response to anticonvulsants is variable, often poor, but the prognosis is favorable. The entity is reported mainly in Asia, and outbreaks are rare in the US and Europe. The above report is the first to show differences in age of onset, the duration of seizures and their response to anticonvulsants, depending on the causative viral infection. In addition to rotavirus, norovirus (Norwalk-like virus) is a significant pathogen in children with $\mathrm{CwG}$. Several different anticonvulsants are generally administered to control the seizure clusters, and none, except perhaps carbamazepine, has demonstrated effectiveness. Trials of antiviral agents might be a more specific and plausible approach to treatment than empiric antiepileptic medications.

\section{SPINAL EXPANDING LESIONS}

\section{EXTRADURAL SPINAL ARACHNOID CYSTS}

A 14-year-old boy with multiple spinal arachnoid cysts and paraplegia, and 37 similar cases in the literature are reviewed by neurosurgeons and radiologist at Univ of Sao Paulo, Brazil. The patient presented with a 4-month history of progressive leg weakness and loss of sensation, clumsiness, and frequent falls. Neurologic exam showed hypoesthesia at T-9 and below, weakness of lower extremities, hyperreflexia, ankle clonus, and Babinski reflexes. MR revealed dorsal compression of the thecal sac with 4 archnoid cysts extending from T-1 to T-9. At surgical dissection and removal, the cyst walls were thickened dura, and the fluid communicated with the CSF. One-year follow-up found no recurrence of cysts and normal ambulation. (de Oliveira RS, Amato MCM, Santos MV et al. Extradural arachnoid cysts in children. Child's Nerv Syst Nov 2007;23:1233-1238). 